\title{
Geographic Information System of Stroke Incidence in Zanjan Province, Iran During 2012-2019
}

\author{
Mohsen Soleimani ${ }^{1 *}$ (D), Ahmad Jalilvand ${ }^{2}$, Roghayeh Soleimani ${ }^{3}$ \\ 1. Dept. of Medical Informatics, Mousavi Hospital, School of Medicine, Zanjan University of Medical Sciences, Zanjan, Iran \\ 2. Dept. of Pathology, Mousavi Hospital, School of Medicine, Zanjan University of Medical Sciences, Zanjan, Iran \\ 3. Dept. of General Psychology, Islamic Azad University, Zanjan Branch, Zanjan, Iran
}

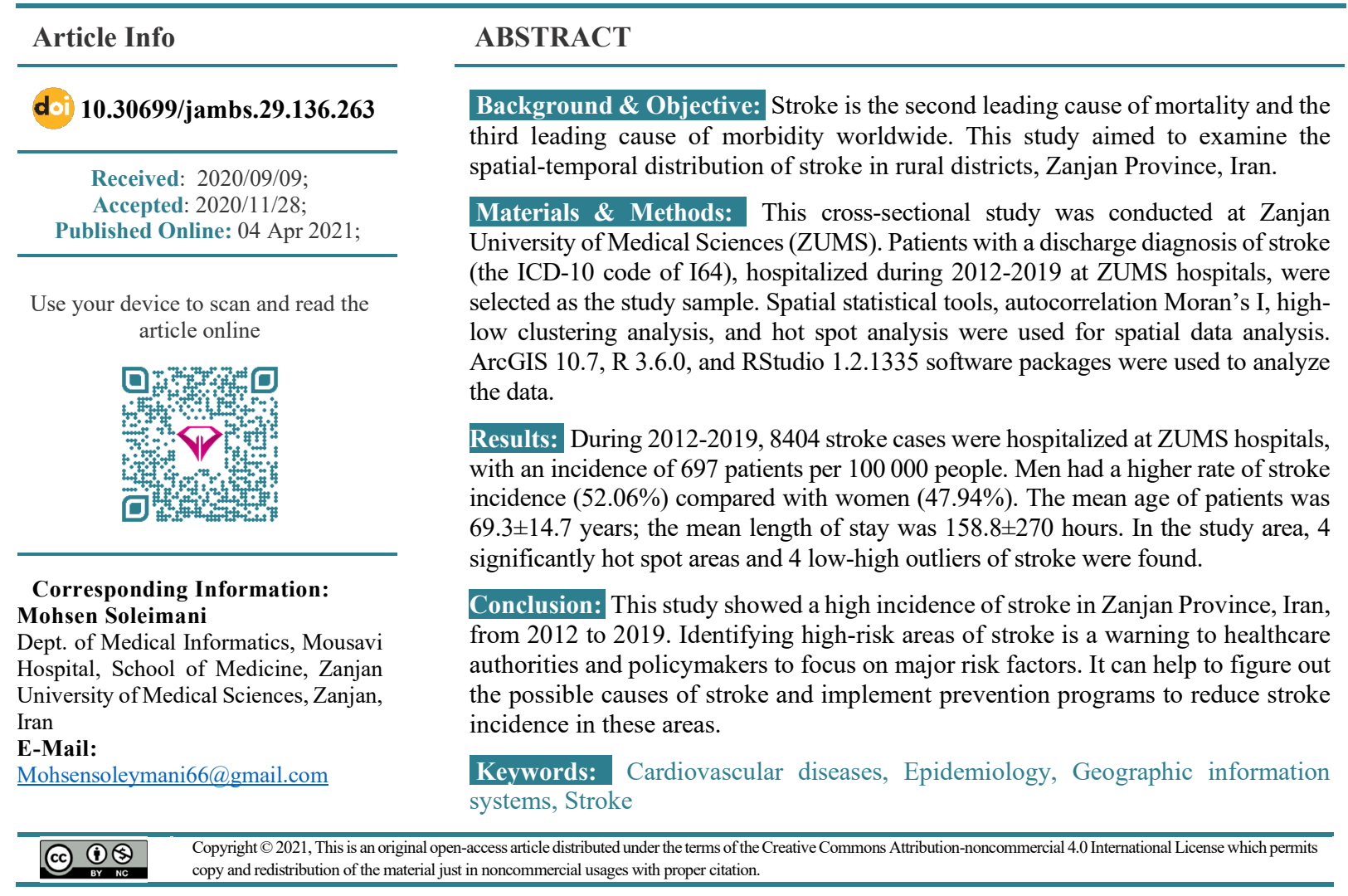

\section{Introduction}

Stroke is the second leading cause of mortality and the third leading cause of morbidity worldwide (1). Stroke is the main cause of 5.5 million deaths globally, of which $70 \%$ of them occur in developing countries (2). The World Health Organization (WHO) defined stroke as "rapidly developing clinical signs of focal (or global) disturbance of cerebral function, with symptoms lasting 24 hours or longer or leading to death, with no apparent cause other than of vascular origin" (3).

The rate of stroke mortality in Iran is higher than in other countries (4). High treatment costs, rehabilitation, and disability are the most important complications of stroke; they impose high annual costs to families and the healthcare system (5).

Previous studies have shown that the incidence of stroke is different in various geographical regions due to risk factors, such as age, sex, and ethnicity $(6,7)$. There are many risk factors that affect the incidence of stroke; they are classified as biological, physiological, lifestyle behaviors, and physical inactivity, as well as social and environmental factors (8).

The incidence of stroke is higher in urban areas than in rural areas, and it increases by changing epidemiological factors and growing elderly population (9). Identifying the main risk factors and implementing preventive programs are the main priorities of the healthcare system to reduce stroke incidence (10).

The famous phrase, "Time is Brain," confirms the importance of diagnosis and treatment of stroke in emergency situations without wasting time. When the stroke progresses, more brain neurons will be destroyed, and more disability will be resulted (11). The spatial analysis of stroke can recognize the spatial clusters and high-risk areas of stroke; it clarifies the relationships between environmental factors and the incidence of stroke in different areas (12). Identifying these areas allows healthcare authorities to plan appropriate intervention 
programs and distribute resources fairly to reduce or prevent the incidence of stroke.

Digitalized maps can increase the quality of decisionmaking and provide $80 \%$ of the information, which decision-makers and policymakers need about geographical locations. Geographic information system (GIS) technology can improve the quality of information and facilitate the decision-making processes in health settings. GIS can be used for gathering, storing, examining, analyzing, and illustrating geographically referenced information $(7,13)$.

Spatial autocorrelation is one of the GIS tools and a method of exploratory spatial data analysis; it allows to detect spatial dependencies or autocorrelations in spatial data (14). There are two main aspects regarding stroke, i.e., (i) treatment and rehabilitation and (ii) the prevention of stroke incidence $(1,4,8)$. Identifying risk factors and geographical distribution of stroke is essential to assess stroke incidence in different areas $(1,6,9,10,13,14)$.

The GIS of stroke generates new knowledge on the spatial variations of stroke, which helps to make decisions about prevention and screening strategies in high-risk areas (12). The reports of stroke occurrence in the province of Zanjan, Iran, were not comprehensive. Zanjan Province is located in the northwest of Iran. It has 8 city districts, and its center is Zanjan City. Its population is 1057461 and covers an area of $291.27 \mathrm{~km}^{2}$ (15). Thus, this study aimed to explore the spatial-temporal variations of stroke incidence in Zanjan Province, Iran, in urban and rural districts during 2012-2019.

\section{Materials and Methods}

This descriptive-analytical study was conducted at Zanjan University of Medical Sciences (ZUMS). Study data included patients' admission's code, national code, residential addresses, ICD-10 discharge diagnosis, admission's date and length of stay; they were collected from 9 hospital information system (HIS) databases. Patients with the ICD-10 discharge diagnosis code of I64 (stroke, not specified as hemorrhage or infarction) were selected as a study sample; they were hospitalized at ZUMS hospitals during 2012-2019.

Spatial statistical tools, Anselin Moran's I, high-low clustering analysis, and hot spot analysis were used for spatial data analysis. The longitude and latitude points of the patients' residence addresses were obtained using Google Maps within a radius of $0.5 \mathrm{~km}$ around their homes. The population of towns and villages was obtained from the Statistics Center of Iran. The stroke incidence rate for each urban and rural district was computed by dividing stroke cases in each district by its population multiplied by 100000 . In this research, urban and rural district boundary defined as the study area; Zanjan Province has 8 urban and 48 rural districts.

Anselin Moran's I statistical autocorrelation tool was used to demonstrate the pattern of stroke distribution. It examines the spatial autocorrelation based on the location of two values and desired geographical features. The results of this analysis determine the distribution pattern of stroke, which is random, dispersed, or clustered. If the Moran index value is close to positive one $(+1)$, the data has a spatial autocorrelation and their distribution pattern is clustered; if the Moran value is close to negative one (1), the distribution pattern of stroke is dispersed (Figure 1).

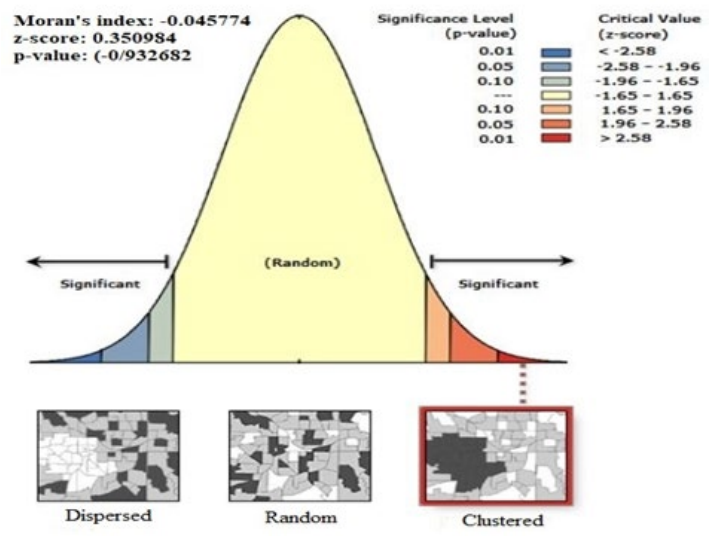

Figure 1. Anselin Moran's I analysis on stroke incidence in Zanjan Province, Iran during 2012-2019

The hot spot analysis calculates the Getis-Ord general $\mathrm{G}$ statistic for all data. This tool detects high-volume side effects (hot spots) and low-volume side effects (cold spots) in a set of weighted data. Hot-spot areas are statistically significant if the areas and their neighborhoods have a high value of stroke incidence rate. Cold-spot areas are statistically significant if the areas and their neighborhoods have a low value of stroke incidence rate.

High-high and low-low clustered regions show that target regions and their neighborhood areas have similar stroke incidence rates. In other words, high-high areas illustrate high-risk areas of stroke, while low-low regions show low-risk areas of stroke. High-low and low-high areas demonstrate the outliers of stroke incidence.

The cluster and outlier analysis was used to confirm and complement the hot-spot analysis; it allows to identify both groupings and areas where anomalies exist. Therefore, its results show aspects that may be ignored in the hot-spot analysis but are important, especially in those areas where different types of groupings coexist. This tool determined spatial clusters of regions with high or low stroke as well as spatial outliers.

Spatial analysis was accomplished using ArcMap 10.7. (ArcGIS, ESRI, Inc., Redlands, California, USA) Descriptive analysis was performed using R 3.6.0 (R Core Team, Vienna, Austria) and RStudio 1.2.1335 (RStudio Team, Boston, MA, USA). The chi-square statistic was used for examining the relationships between categorical variables. This study was approved by the Ethics Committee of ZUMS (Code IR.ZUMS.REC.1398.056). 


\section{Results}

Among a total of 8974 registered stroke cases, patients from other provinces or with incomplete residential addresses were excluded (570 cases); among these, 8404 cases were confirmed with the occurrence of 697 cases per 100000 people. The incidence of stroke was higher in men $(52.06 \%)$ than in women (47.94\%). The mean age of stroke patients was $69.3 \pm 14.7$ years, and the mean length of stay was $158.8 \pm 270$ hours.

The highest incidence of stroke rate was in Ijrood City with 916 cases per 100000 people; the lowest incidence was in Abhar City with 347 cases per 100000 people (Table 1).

Table 1. Stroke incidence rates by city in Zanjan province, Iran, from 2012-2019

\begin{tabular}{|cccccccc} 
County & Population & $\begin{array}{c}\text { Total } \\
\text { N }(\%)\end{array}$ & $\begin{array}{c}\text { Women } \\
\mathbf{N}(\%)\end{array}$ & $\begin{array}{c}\text { Men } \\
\mathbf{N}(\%)\end{array}$ & $\begin{array}{c}\text { Per } \\
\mathbf{1 0 0 , 0 0 0}\end{array}$ & $\begin{array}{c}\text { Age } \\
\text { AVG } \pm \text { SD }\end{array}$ & $\begin{array}{c}\text { LOS } \\
\text { AVG } \pm \text { SD }\end{array}$ \\
\hline Zanjan & 584159 & $5281(62.8)$ & $2548(48.25)$ & $2733(51.75)$ & 904 & $68.1 \pm 15.5$ & $142.8 \pm 232.2$ \\
\hline Abhar & 167824 & $582(6.9)$ & $253(43.47)$ & $329(56.53)$ & 347 & $68.9 \pm 15.6$ & $148.1 \pm 248.7$ \\
\hline Khoramdareh & 77443 & $294(3.5)$ & $149(50.68)$ & $145(49.32)$ & 379 & $68.9 \pm 14.8$ & $160.2 \pm 586.3$ \\
\hline Khodabandeh & 195741 & $973(11.6)$ & $477(49.02)$ & $496(50.98)$ & 497 & $68.2 \pm 14.2$ & $174.2 \pm 250.5$ \\
\hline Soltanieh & 35118 & $232(2.8)$ & $108(46.55)$ & $124(53.45)$ & 661 & $71.5 \pm 14.4$ & $161.1 \pm 189.5$ \\
\hline Tarom & 54656 & $329(3.9)$ & $161(48.94)$ & $168(51.06)$ & 602 & $70.6 \pm 14.5$ & $161.7 \pm 187.6$ \\
\hline Ijrood & 43795 & $401(4.8)$ & $191(47.63)$ & $210(52.37)$ & 916 & $69.2 \pm 15$ & $154.1 \pm 217.1$ \\
\hline Mahneshan & 46484 & $312(3.7)$ & $142(45.51)$ & $170(54.49)$ & 671 & $69.1 \pm 13.7$ & $168.4 \pm 247.8$ \\
\hline Total & 1205220 & $8404(100.0)$ & $4029(47.94)$ & $4375(52.06)$ & 697 & $69.3 \pm 14.7$ & $158.8 \pm 270$ \\
\hline
\end{tabular}

The GIS of stroke showed that the incidence of stroke was more prevalent in the northern and central cities of the study area (Figure 1). The occurrence rate per 100000 people was increased in all rural districts during 2012-2019 (Figure 2). Age distribution of stroke showed that the age group of 50-60 years and older were the higher risk age group for stroke incidence $(P<0.0001$; see Figure 3$)$. The results of the temporal analysis showed that the incidence of stroke rate increased significantly from 409 cases in 2012 to 1750 cases in 2019 per 100000 people in Zanjan Province; the mean length of stay of stroke decreased from 165.5 hours in 2012 to 120.8 hours in 2019 (Figure 3).

According to the numerical output of Anselin Moran's statistical analysis, the distribution of stroke incidence was not in the confidence range; it was not statistically significant (Moran's index: -0.045774). Therefore, there was no spatial autocorrelation between the patterns of stroke distribution; it was random (Figure 1).

Figure 4 reveals the hot-spot analysis of stroke distribution. The red areas indicate large amounts of Zvalue in the hot-spot analysis; the small and negative $\mathrm{Z}$-values make the blue areas (cold spots). It showed that there were significantly 4 hot spots in Taham, Gilvan, Qarabolaq, and Gozaldareh rural districts. However, there was not any significant cold spot in the study area.
The spatial cluster analysis of strokes showed that there was 1 significant low-low outlier of stroke incidence in the Anguran rural district. Further, there were also 4 significant low-high outliers of stroke incidence in the eastern and central cities of the study area (Figure 5). 


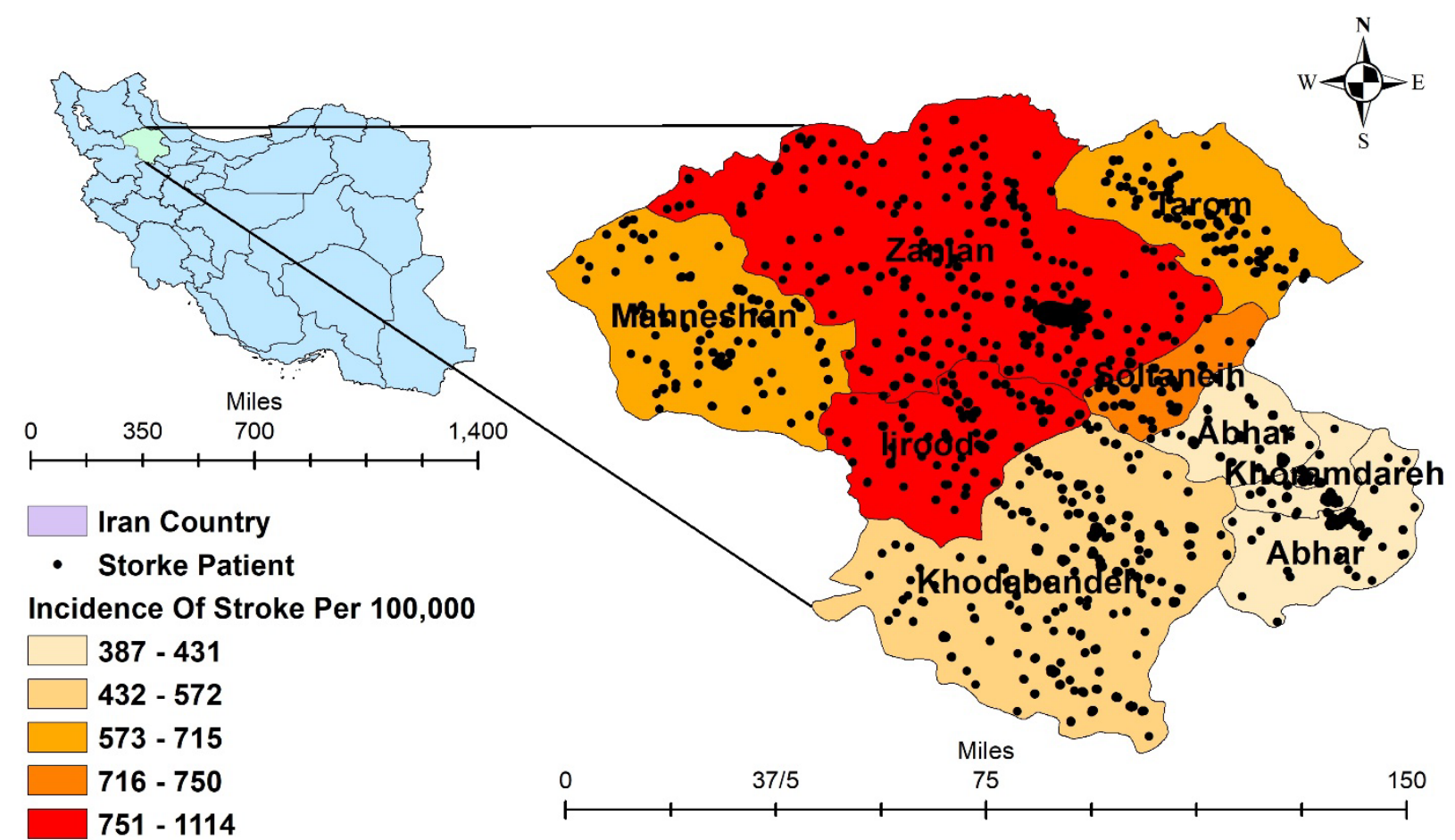

Figure 2. Geographical distribution of stroke patients at county level in Zanjan Province, Iran during 2012-2019

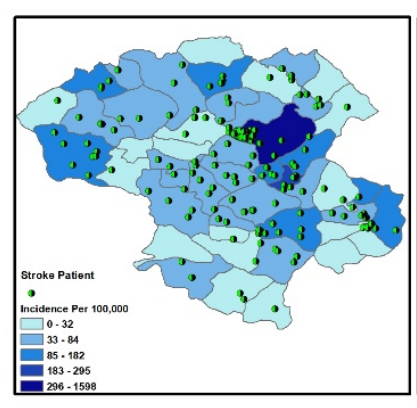

2012-2013

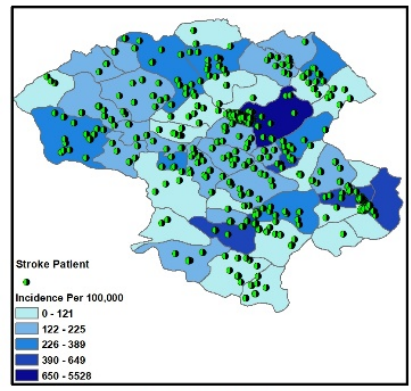

2016-2017

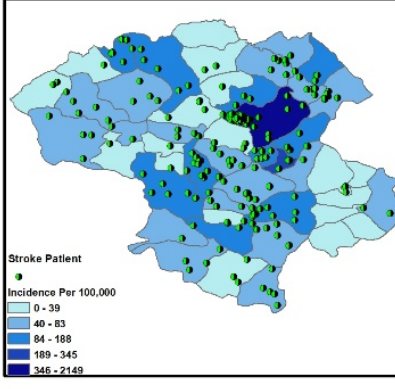

2013-2014

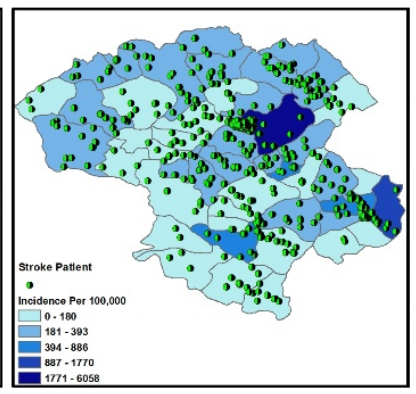

2017-2018

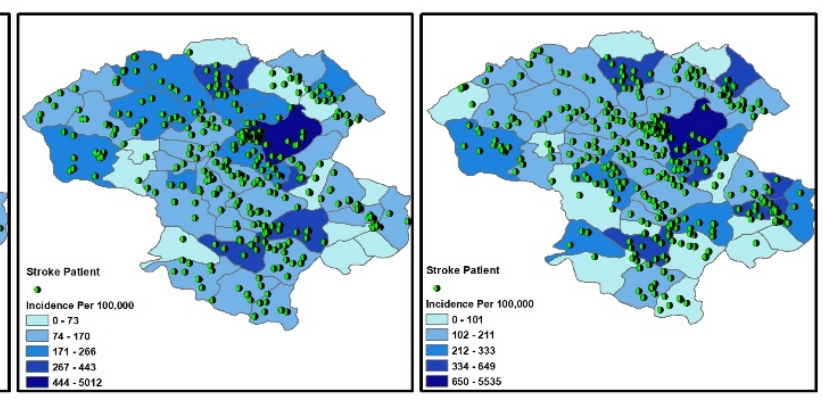

2014-2015

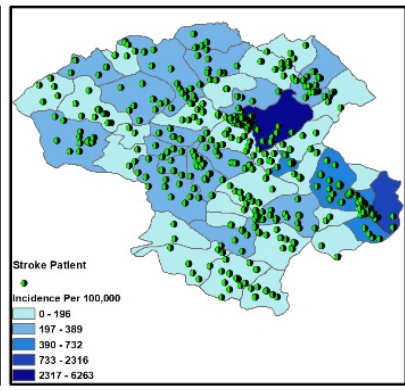

2018-2019

Figure 3. Geographical distribution of stroke patients at the rural district level during 2012-2019 in Zanjan Province, Iran 

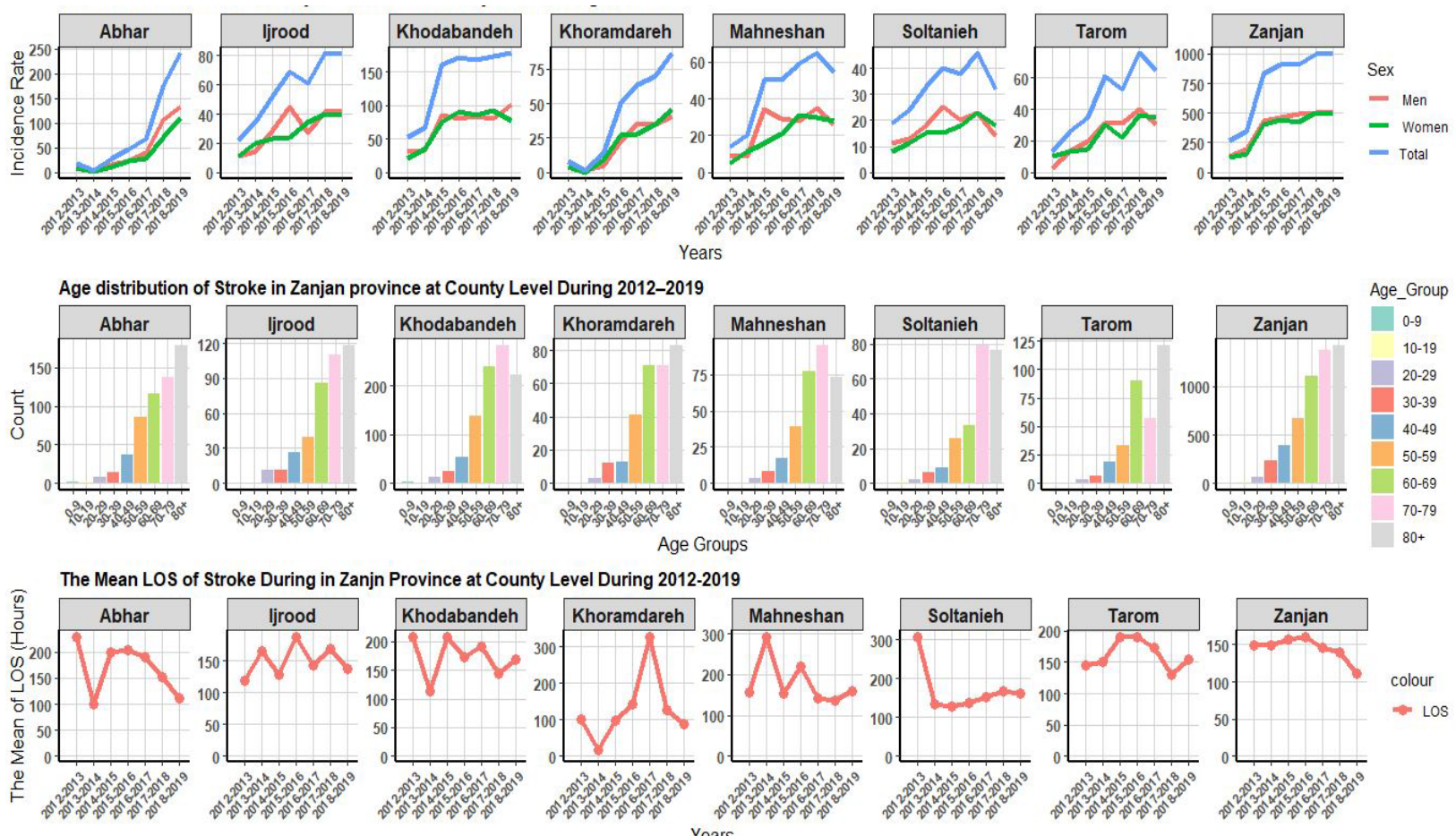

Figure 4. The incidence rate per 100,000, age distribution and the mean LOS of stroke in Zanjan province, Iran during 2012-2019
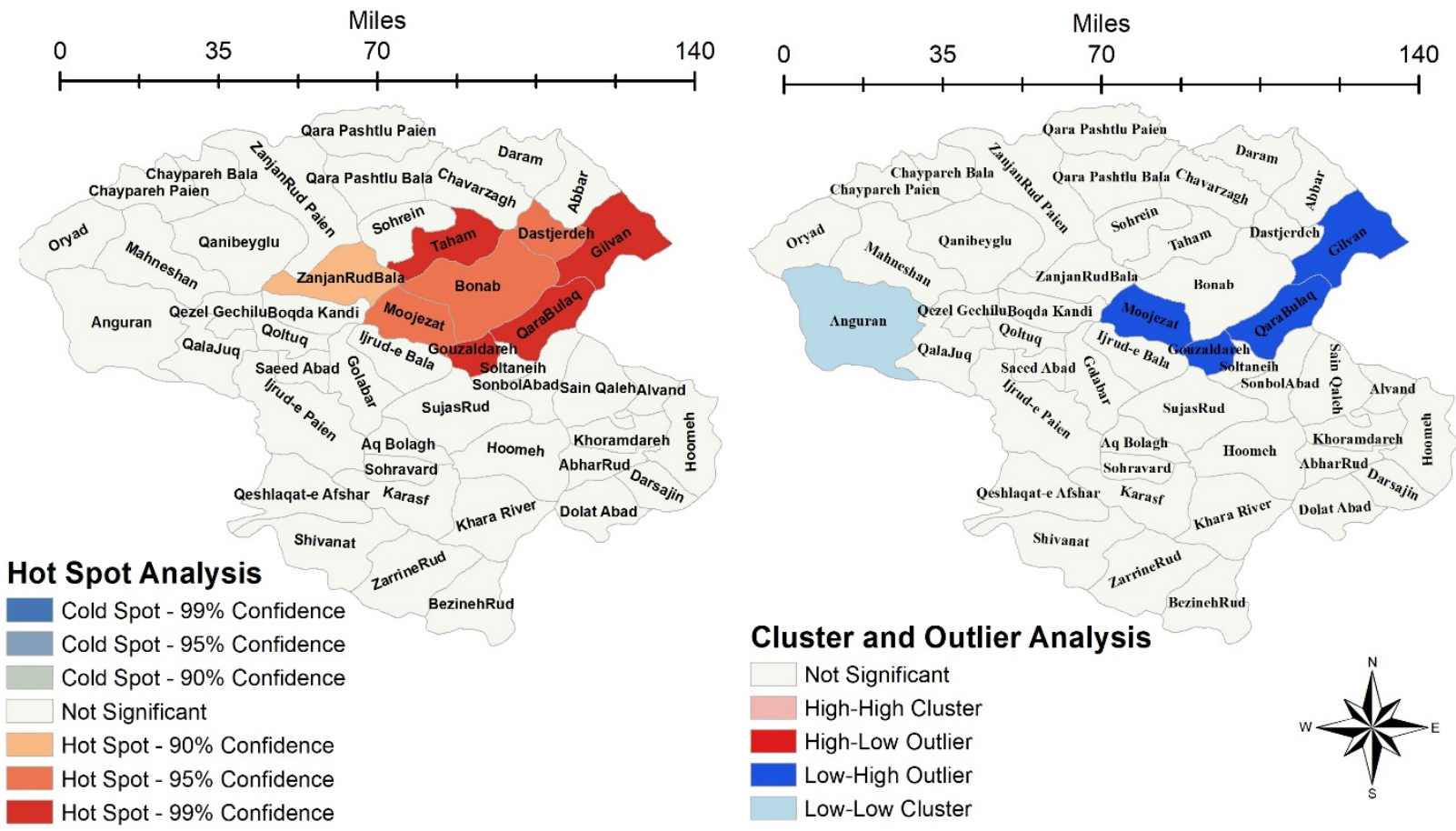

\section{Cluster and Outlier Analysis}
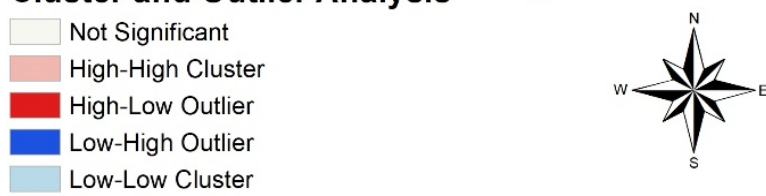

Figure 5. Hotspot and High-Low clustering analysis of stroke in rural district level in Zanjan province, Iran during 2012-2019 


\section{Discussion}

This study was the first geospatial and temporal analysis of stroke incidence, conducted in Zanjan Province, Iran. The results of this study showed that the incidence of stroke rate was higher in men than in women, which is consistent with the results of studies conducted in Europe (16), the United States (17), and Arab countries (18). These differences may be due to the physiological differences between the two genders.

Aging is the most important risk factor; the incidence of stroke almost doubles after the age of 55 (17). In this study, the incidence of stroke was lower in patients under 50 years old, but it increased in the sixth and seventh decades of life (Figure 3). However, it was higher in the ninth decade of life in Europe (16) and the USA (17), which is inconsistent with the results of this study. Previous studies have shown that the incidence of stroke was higher in the urban population than in the rural population (9); it was consistent with the result of this study. The greatest incidence rate of stroke was in Zanjan and Ijrood Cities.

Various risk factors involved in stroke, such as biological, physiological, lifestyle behaviors, and physical inactivity, as well as social and environmental factors $(5,8)$. Different climates and seasons also affect the geographical variation of stroke incidence. The highest incidence of stroke was in the fall season $(33 \%)$, while the lowest was in the summer $(P<0.0001)$ (19). According to the results, for reducing the incidence of stroke rate, this study recommended two approaches as follows: (i) defining and monitoring the major risk factors in high-risk areas and (ii) monitoring these risk factors in people over the age of 50 .

Spatial analysis of stroke distribution plays a crucial role in visualizing and predicting the spatial variation of stroke patterns in different areas. There are two important types of spatial statistic tools for identifying geographic changes in stroke incidence rates. Global methods are more sensitive to the null hypothesis; they can illustrate spatial structures but are not able to identify the clusters' situation. Local cluster statistics quantify spatial autocorrelation and clustering at the small area level $(13,20)$.

As the aim of this study was to examine the spatialtemporal variations of stroke incidence in Zanjan Province (Iran) and also identify the spatial clusters of stroke incidence in rural district levels, Anselin Local Moran's I was an appropriate statistical tool; it was used to analyze the local clustering of stroke in this study.

The hot-spot analysis tools recognize statistically significant spatial clusters of high values (hot spots) and low values (cold spots) (20). The distribution of stroke incidence patterns in the province of Zanjan was random; there were just 4 hot spot areas in this study. The results of high-low clustering analysis also confirmed the results of Anselin Moran's I statistic; also, there were just 4 outliers of stroke incidence in the study area. The results of a study conducted in the USA showed significant spatial clustering at the neighborhood level of cities, both in and outside of the stroke belt and also within all areas with above 1 million occupants; this finding is inconsistent with the results of this study (12).

Many reasons, such as environmental factors, demographics, economic and social status, health literacy, availability of medical centers, etc., play a role in creating stroke's spatial clusters $(6,9,12)$. Although stroke has been recognized as a time-sensitive condition (which needs immediate therapeutic interventions), significant barriers still remain in rapid and effective treatment (21). Knowledge about the spatial distribution of stroke can help the emergency departments and healthcare authorities to identify highrisk areas to optimize the deployment of emergency resources to reduce stroke incidence. It is recommended to use the results of this study in future works to identify and monitor important risk factors of stroke and optimize the deployment of emergency resources at high-risk areas.

\section{Limitations}

This research had some limitations. First, this study was defined by stroke patients admitted to the ZUMS hospitals in the province of Zanjan, Iran. Thus, the patients may not be representative of all stroke patients in Zanjan hospitals. The patients of two social security organizations' hospitals (such as Tamin Ejtemaee) did not include in the study. Another limitation was the use of census population data as the dominant method for calculating stroke rates. The population census is conducted every 5 years in Iran. Due to the absence of an annual population census, this study used the census data of 2011-2016; by increasing the number of births and reducing the number of deaths per year, the population of each year (2012-2019) was estimated.

\section{Conclusion}

The results of this study showed that the incidence of stroke rate increased in recent years in Zanjan Province, Iran. There was no spatial autocorrelation between the incidence of stroke and its distribution; it was random, while several regions in the center and west of the study area were identified as stroke highrisk areas. Identifying high-risk areas of stroke is a warning to the healthcare authorities and policymakers to focus on major risk factors; it helps them to figure out the possible causes of stroke and implement prevention programs to reduce stroke incidence rate in these areas. 


\section{Acknowledgments}

This study was a part of a Health Systems Research (HSR). It was financially supported by Zanjan University of Medical Sciences (ZUMS). (Code A-121171-3).

\section{Ethical Approval and Consent to Participate}

This study was approved by the Ethics Committee of ZUMS (Code IR.ZUMS.REC.1398.056).

\section{Availability of Data and Material}

The data set generated and / or analyzed in this study is not available to the public, due to the use of this data for other research studies, which have not been published, yet.

\section{Funding}

This study was financially supported by Health Systems Research (HSR) committee of ZUMS.

\section{Conflict of Interest}

The authors declare that they have no conflicts of interests.

\section{References}

1. Johnson CO, Nguyen M, Roth GA, et al. Global, regional, and national burden of stroke, 19902016: a systematic analysis for the Global Burden of Disease Study 2016. Lancet Neurol. 2019;18(5):439-58. [DOI:10.1016/S14744422(19)30034-1]

2. Farhoudi M, Mehrvar K, Sadeghi-Bazargani H, et al. Stroke subtypes, risk factors and mortality rate in northwest of Iran. Iran J Neurol. 2017;16(3):112-7.

3. Mazaheri S, Beheshti F, Hosseinzadeh A, Mazdeh M, Ghiasian M. Epidemiologic study of cardinal risk factors of stroke in patients who referred to Farshchian hospital of Hamadan during 2014-2015. Avicenna J Clin Med. 2016;22(4):331-7.

4. Borhani-Haghighi A, Safari R, Heydari ST, et al. Hospital mortality associated with stroke in southern iran. Iran J Med Sci. 2013;38(4):31420 .
5. Sridharan SE, Unnikrishnan JP, Sukumaran S, et al. Incidence, types, risk factors, and outcome of stroke in a developing country: the Trivandrum Stroke Registry. Stroke. 2009;40(4):1212-8. [DOI: 10.1161/STROKEAHA. 108.531293]

6. Mungrue K, Saroop K, Samsundar A, et al. The epidemiology and spatial analysis of stroke in Trinidad and Tobago in the first decade of the $21^{\text {st }}$ century (2000-2009). Health. 2014;06:72937. [DOI: 10.4236/health.2014.68094]

7. Soleimani M, Jalilvand A, Soleimani R. Geographical epidemiology of cardiovascular diseases in Zanjan province: analysis of groups from the international classification of diseases, 10th Revision. J Adv Med Biomed Res. 2020;28(127):90-6. [DOI: 10.30699/jambs.28.127.90]

8. Boehme AK, Esenwa C, Elkind MSV. Stroke risk factors, genetics, and prevention. Circ Res. 2017;120(3):472-95.

[DOI: 10.1161/CIRCRESAHA.116.308398]

9. Engstrom G, Jerntorp I, Pessah-Rasmussen H, Hedblad B, Berglund G, Janzon L. Geographic distribution of stroke incidence within an urban population: relations to socioeconomic circumstances and prevalence of cardiovascular risk factors. Stroke. 2001;32(5):1098-103. [DOI: 10.1161/01.STR.32.5.1098]

10. Rheenen S, Watson T, Alexander S, Hill M. An analysis of spatial clustering of stroke types, inhospital mortality, and reported risk factors in Alberta, Canada, using geographic information systems. Can J Neurol Sci. 2015;42:1-11. [DOI:10.1017/cjn.2015.241]

11. Neuhaus AA, Couch Y, Hadley G, Buchan AM. Neuroprotection in stroke: the importance of collaboration and reproducibility. Brain. 2017;140(8):2079-92. [DOI:10.1093/brain/awx126]

12. Dworkis DA, Marvel J, Sanossian N, Arora S. Neighborhood-level stroke hot spots within major United States cities. Am J Emerg Med. 2020. [DOI:10.1016/j.ajem.2019.06.044]

13. Sánchez Martín JM, Rengifo Gallego J, Morato R. Hot spot analysis versus cluster and outlier analysis: An enquiry into the grouping of rural accommodation in Extremadura (Spain). Int $\mathbf{J}$ Geo-Inform. 2019;8:176. [DOI: 10.3390/ijgi8040176] 
14. Khan D, Rossen LM, Hamilton BE, He Y, Wei $\mathrm{R}$, Dienes E. Hot spots, cluster detection and spatial outlier analysis of teen birth rates in the U.S., 2003-2012. Spat Spatiotemporal Epidemiol. 2017;21:67-75. [DOI:10.1016/j.sste.2017.03.002]

15. Iran SCo. IRAN Statistical yearbook 20162017. Tehran: Statistical Center of Iran; 2017.

16. Putaala J, Yesilot N, Waje-Andreassen U, et al. Demographic and geographic vascular risk factor differences in European young adults with ischemic stroke the 15 cities young stroke study. Stroke. 2012;43:2624-30. [DOI:10.1161/STROKEAHA. 112.662866]

17. Avan A, Digaleh H, Di Napoli M, et al. Socioeconomic status and stroke incidence, prevalence, mortality, and worldwide burden: an ecological analysis from the Global Burden of Disease Study 2017. BMC Med. 2019;17(1):191. [DOI:10.1186/s12916-0191397-3]

18. Farghaly W, El-Tallawy H, Shehata G, et al. Epidemiology of nonfatal stroke and transient ischemic attack in Al-Kharga District, New
Valley, Egypt. Neuropsychiatr Dis Treat. 2013;9:1785-90. [DOI:10.2147/NDT.S48322]

19. Hosininezhad M, Bakhshayesh B , Moaddabi Y, Hatamyan HR .Investigating the seasonal pattern of stroke incidence and the association between daily stroke occurrences and meteorological factors. J Guilan Univ Med Sci. 2014;23(90):50-8.

20. Jacquez G. Spatial Cluster Analysis. The handbook of geographic information science: S. Fotheringham and J. Wilson. Blackwell Publishing; 2005.

21. Rajan S, Baraniuk S, Parker S, Wu T-C, Bowry $\mathrm{R}$, Grotta J. Implementing a mobile stroke unit program in the United States: Why, how, and how much? JAMA Neurol. 2015; 72(2):229-34 [DOI:10.1001/jamaneurol.2014.3618]

\section{How to Cite This Article:}

Soleimani M, Jalilvand A, Soleimani R. Geographic Information System of Stroke Incidence in Zanjan Province, Iran During 2012-2019. J Adv Med Biomed Res. 2021; 29 (136) :263-270

\section{Download citation:}

$\underline{\text { BibTeX }}|\underline{\text { RIS }}| \underline{\text { EndNote }}|\underline{\text { Medlars }}| \underline{\text { ProCite }}|\underline{\text { Reference Manager }}| \underline{\text { RefWorks }}$

\section{Send citation to:}

Mendeley 2 Zotero ; RefWorks RefWorks 